\title{
Scintimetric Characterization of Skeletal Hotspots by Dr.V.Siva's Retention Ratio in A Diagnostic Referral Center
}

\author{
Sivasubramaniyan $\mathbf{V}^{1,2 *}$ and $\mathbf{K}$ Venkataramaniah ${ }^{3}$ \\ ${ }^{1}$ Doctoral Research Student, Department of Physics, India \\ ${ }^{2}$ Senior Consultant Nuclear Medicine Physician, Vijaya Health Centre, India \\ ${ }^{3}$ Senior Professor, SSSIHL, Prasanthinilayam, India
}

Received: 制 June 25, 2018; Published: 啙 July 16, 2018

*Corresponding author: Sivasubramaniyan V, Doctoral Research Student, Department of Physics, SSSIHL, Senior Consultant Nuclear Medicine Physician, Vijaya Nuclear Scans \& Therapy, Vijaya Health Centre, Vadapalani Chennai, India

\section{Abstract}

Aim: This study aims to document the utility of Scintimetric Characterization of the Skeletal Hot Spots seen in the Bone Scans done in this referral center during 2016-2017, in differentiating metastatic and non-metastatic lesions.

Materials and Method: The study group consists of 59 patients referred for metastatic evaluation and non-specific bone complaints to our center. The whole body bone scan was done $3 \mathrm{hrs}$ after the intravenous injection of 15 to $25 \mathrm{mCi}$ of Tc99m MDP using GE Millenium Gamma Camera. The $24 \mathrm{hr}$ whole body bone scan was repeated in all those who had focal hot spots in their scans and who accepted voluntarily with the consent of the referring physician. The counts in the focal hot spots at 3 and $24 \mathrm{hr}$ bone scan images were calculated using the region ratio count protocol. The Dr.V.Siva's Retention Ratio was calculated by dividing the $3 \mathrm{hr}$ counts by the $24 \mathrm{hr}$ counts in the focal hotspots. The values of metastatic and nonmetastatic groups were tabulated and analyzed.

Results: In our series 35/59 (59\%) scans were negative and did not show any focal changes. 24/59 (41\%) scans showed focal changes in the form of hot spots. Out of the total 69 hot spots analyzed $31(45 \%)$ had the value of $13.07 \pm 1.02$ indicating metastatic nature and 38 ( $55 \%$ ) had the value of $6.07 \pm 0.62$ confirming the benign nature of the lesions. Thus the metastatic group had the mean value which is almost twice that of the benign group. The statistical evaluation confirmed the significant difference between the two groups.

Conclusion: It can be concluded that the Scintimetric characterization of the focal hot spots by Dr.V.Siva's retention ratio effectively provides useful means to differentiate between the metastatic and non-metastatic lesions in the tertiary referral diagnostic center as well.

Keywords: Scintimetry; Dr.V Siva's Retention Ratio; Skeletal Hot Spot Characterization; Differentiation

\section{Introduction}

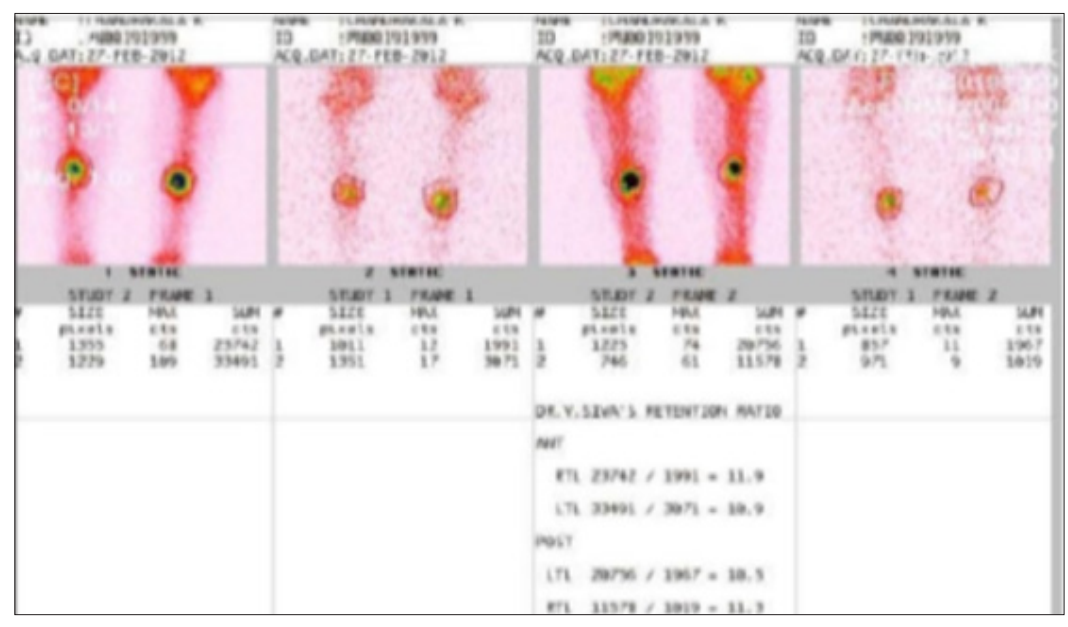

Figure 1: Dr. V. Sivas Retention Ratio Calculation.

The whole-body bone scan is usually asked for the skeletal metastatic involvement in the known and proven cases of Carcinoma
Breast, Carcinoma Prostate and Carcinoma of the Thyroid and lung most of the time and in other tumors as and when indicated. The 
normal bones show normal uptake and uniform distribution of the tracer. The abnormal regions usually present as focal hot spots due to increased localization of the tracer in the affected bone. This is common to both the neoplastic and benign etiologies. The metastatic lesions tend to be multiple, asymmetric in distribution and can be single as well. The benign lesions tend to be solitary and if multiple normally show symmetrical distribution of the tracer. The utility of $24 \mathrm{hr}$ delayed bone scan in differentiating the benign and neoplastic conditions was first shown by Israel 0 et al. [1]. The counts at the lesion are dived by the counts at the background at $4 \mathrm{hr}$ scan and the $24 \mathrm{hr}$ scan. The ratio of the $24 \mathrm{hr}$ lesion / nonlesion value divided by the $4 \mathrm{hr}$ lesion / nonlesion value was shown to be different in the Malignant and benign bone disorders. This method proved to be of no clinical value as the resultant ratio was in decimal values and no useful cut off could be arrived at. Therefore by taking the counts only in the lesion at $4 \mathrm{hr}$ and $24 \mathrm{hr}$ Dr. V.Siva's retention ratio was calculated by dividing the $4 \mathrm{hr}$ count by $24 \mathrm{hr}$ count as shown in Figure 1. A useful cut off could be arrived at using this ratio as it gave full integers rather than decimal values $[2,3]$. The utility of this method in the evaluation of skeletal hot spots in the

Table 1: Malignant group Data.

\begin{tabular}{|c|c|c|c|}
\hline SITE & $4 \mathrm{hr}$ Count & $24 \mathrm{hr}$ Count & Dr. V Sivas Ratio \\
\hline D5 & 8295 & 541 & 15.3 \\
\hline D6 & 11244 & 837 & 13.4 \\
\hline L1 & 10929 & 583 & 11.5 \\
\hline L2 & 8291 & 1139 & 14.2 \\
\hline D10 & 15008 & 1277 & 13.1 \\
\hline L4 & 17239 & 2105 & 13.4 \\
\hline L5s & 19196 & 1604 & 9.1 \\
\hline S1 & 18339 & 1474 & 11.4 \\
\hline LTSIJ & 18374 & 645 & 12.4 \\
\hline \multirow{2}{*}{ Rt Rib } & 6149 & 1213 & 9.5 \\
\hline & 15185 & 604 & 12.5 \\
\hline Rt & 4495 & 357 & 11 \\
\hline Scap & 3757 & 461 & 10.5 \\
\hline rib & 4421 & 438 & 9.5 \\
\hline RIB & 5620 & 1430 & 12.8 \\
\hline 8thRib & 26709 & 1327 & 18.6 \\
\hline \multirow{2}{*}{ Rt.Sho } & 16452 & 1582 & 12.3 \\
\hline & 21823 & 190 & 13.7 \\
\hline D6 & 4053 & 204 & 21.3 \\
\hline RT 4th & 2337 & 545 & 11.4 \\
\hline
\end{tabular}

Table 2: Benign group Data.

\begin{tabular}{|c|c|c|c|}
\hline SITEL2 & 4hr & 24hr Count & Dr. V Siva's Ratio \\
\hline D6 & 25938 & 3394 & 7.6 \\
\hline L2 & 23345 & 2655 & 7.7 \\
\hline Lt SIJ & 57560 & 7713 & 7.4 \\
\hline D6 & 18930 & 4152 & 4.5 \\
\hline L2 & 13952 & 2107 & 6.6 \\
\hline
\end{tabular}

Cite this article: Sivasubramaniyan V. K Venkataramaniah . Scintimetric Characterization of Skeletal Hotspots by Dr.V.Siva's Retention Ratio in A Diagnostic Referral Center. Biomed J Sci\&Tech Res 6(5)- 2018. BJSTR. MS.ID.001420. DOI: 10.26717/ BJSTR.2018.06.001420. 


\begin{tabular}{|c|c|c|c|}
\hline \multirow{2}{*}{ LT.HIP } & 46865 & 5113 & 9.1 \\
\cline { 2 - 4 } & 54889 & 8272 & 6.6 \\
\cline { 2 - 4 } & 39346 & 5488 & 7.1 \\
\hline \multirow{2}{*}{ D8 } & 89315 & 15214 & 5.8 \\
\hline Lt sca & 26003 & 5016 & 5.1 \\
\hline Rt Ulna & 26253 & 13843 & 1.8 \\
\hline Lt Ulna & 1607 & 365 & 4.4 \\
\hline Low Fem & 1704 & 452 & 3.7 \\
\hline MS FEM & 1786 & 424 & 4.2 \\
\hline RT4RIB & 26501 & 8817 & 3 \\
\hline RT5RIB & 1478 & 393 & 3.7 \\
\hline RT6RIB & 2516 & 547 & 4.5 \\
\hline LT5RIB & 2804 & 629 & 4.4 \\
\hline LT8RIB & 1948 & 531 & 3.6 \\
\hline \multirow{2}{*}{ Lt Ank } & 2116 & 464 & 4.5 \\
\hline
\end{tabular}

\section{Results}

In this series 35 35/59 (59\%) scans were negative and did not show any focal changes. $24 / 59$ (41\%) scans showed focal changes in the form of hot spots. Out of the total 69 hot spots analyzed $31(45 \%)$ had the value of $13.07 \pm 1.02$ indicating metastatic nature and $38(55 \%)$ had the value of $6.07 \pm 0.62$ confirming the benign nature of the lesions as per the Dr. V Siva's retention ration calculation method. Thus the metastatic group had the mean value which is almost twice that of the benign group. The statistical evaluation confirmed the significant difference between the two groups as shown in Figure 2. The results of an unpaired ttest performed showed $\mathrm{t}=12.3$ and $\mathrm{std} \mathrm{dev}=2.35$, degrees of freedom $=67$. The probability of this result, assuming the null hypothesis, is less than .0001 . The test of two independent sample analysis by equal variance method showed that Mean a- Mean b is 7.0037 with t of +12.3 , df 67 and $p$ value of $<0.0001$ by both one tailed and two tailed methods. Similarly in the unequal variance method the Mean a- Mean b is 7.0037 with t of 11.88 , df 51.05 and $p$ value of $<0.0001$ by both one tailed and two tailed methods.

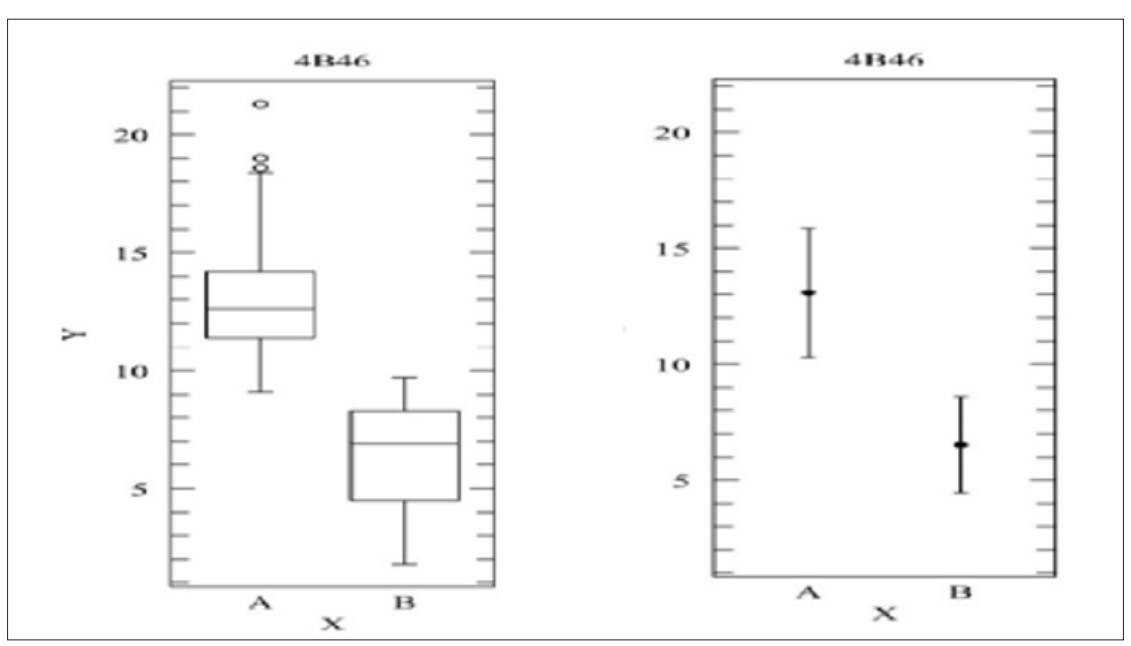

Figure 2: FStudent $\mathrm{T}$ test results.

\section{Discussion}

The scitimetric characterization of the skeletal hot spots had been shown to be useful in the differentiation of benign and malignant lesions and also in the evaluation of delayed union of fractures as well by us [5]. Afroza Naznin et.al from INMAS, Bangladesh in their study have reported that the Dr. V. Siva's retention ratio protocol applied on a small number of prostate cancer patients in Bangladesh gave promising results [6]. They had concluded that the inclusion of Dr. V Siva's quantitative parameter in adjunct to conventional bone scintigraphy for the skeletal survey of carcinoma prostate patients can help to narrow down the specificity spectrum of bone scan hot spots being either due to metastasis versus benign disease. A typical method of reporting the scintimetric characterization was introduced in the report of the study as shown in Figure 3. 


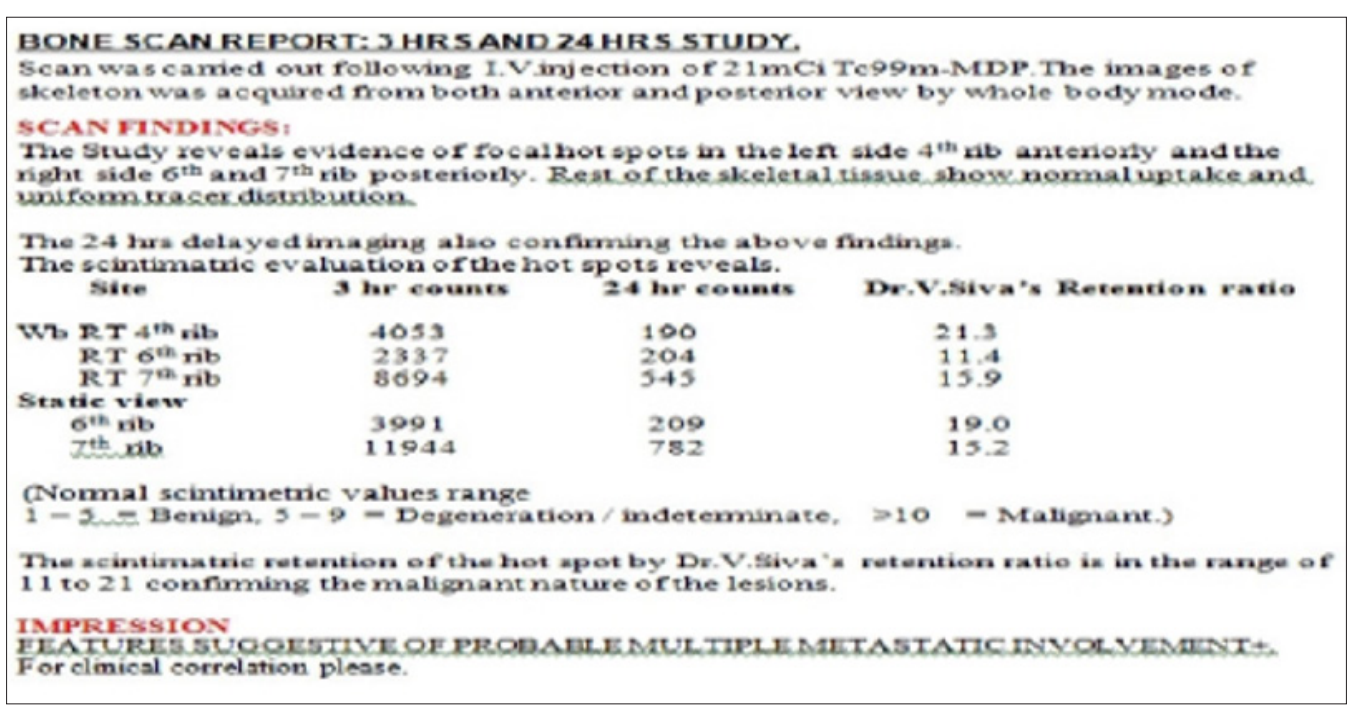

Figure 3: Report Sample.

\section{Conclusion}

It can be concluded that the Scintimetric Characterization of the Skeletal Hot spots by Dr.V. Siva'sRetention Ratio significantly helps in the proper management of the patients even in a tertiary diagnostic center too. This concept must be put into test in various institutions world over for further authentication.

\section{References}

1. Israel O, Front D, Frenkel A, Kleinhaus U (1985) 24 hour / 4 hour ratio of technetium 99m methylene diphosphonate uptake in patients with bone metastases and degenerative bone changes. J Nucl Med 26(3): 237-240.

2. Sivasubramaniyan V, Venkataramaniah K (2015) Scintimetric Characterization of Skeletal Hot Spots in Carcinoma Prostate. I-PET 2015, IAEA-CN-232/136

\section{ISSN: 2574-1241}

DOI: $10.26717 / B J S T R .2018 .06 .001420$

Sivasubramaniyan $\boldsymbol{V}$. Biomed J Sci \& Tech Res

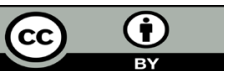

This work is licensed under Creative Commons Attribution 4.0 License

Submission Link: https://biomedres.us/submit-manuscript.php
3. Sivasubramaniyan V, Venkataramaniah K (2016) Temporal Scintimetric Characterization of Skeletal Hotspots in Bone Scan by Dr. V. Siva's Retention Ratio , S.C. Satapathy et al. In: Sivasubramaniyan V, Venkataramaniah K (Eds.), Information Systems Design and Intelligent Applications, Advances in Intelligent Systems and Computing 433: 297305.

4. Sivasubramaniyan V, Venkataramaniah K (2016) Non-invasive QuantitativeCharacterization of Skeletal Metastasis in Carcinoma Prostate by Tc99m MDP Bone Scans Using Dr. V. Siva's Retention Ratio in Correlation with Serum PSA Levels. Med Surg Urol 5: 2.

5. Rahul Namdeo, Sivasubramaniyan V, Venkataramaniah K (2015) Scintimetric Evaluation in the Assessment of Delayed Union of SkeletalFractures.International Journal of Innovative Research \& Development 4(8): 362-365.

6. Afroza Naznin, Sadia Sultana, Amrun Nahar, Taslima Sifat, Pupree Mutsuddy (2017) Evaluation of Skeletal Hot Spots in Carcinoma Prostate Patients by Scintimetric Method. Bangladesh J Nucl Med 20(1): 9-13.

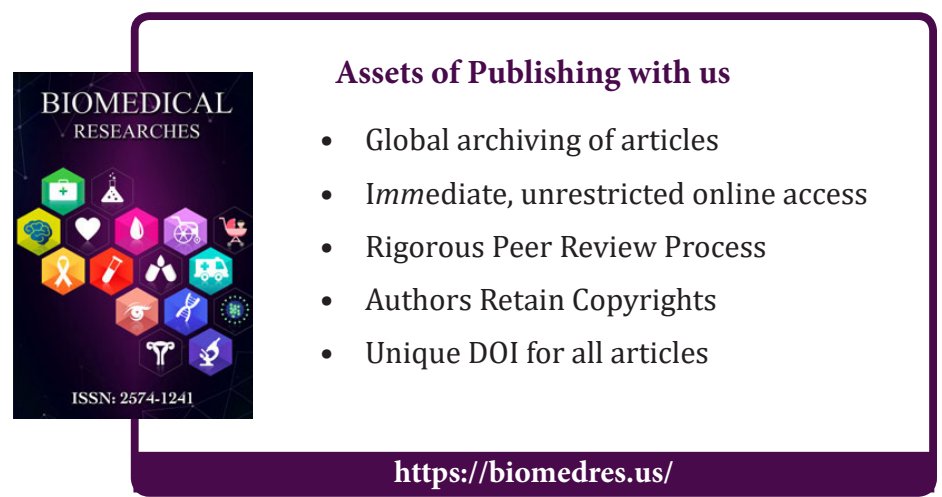

